\title{
Pinturas a base de cal: revisión del mercado y acotación normativa
}

\author{
Lime-based paints: market review and regulatory \\ assessment
}

\begin{abstract}
Brenda Levano
Antonia Navarro Ezquerra Joan Ramon Rosell Amigó

\section{Resumen}

finales de los años 70 la sociedad comenzó a tomar conciencia de los A peligros de la pintura sintética con respecto a la salud y a la

tradicionales "saludables" y que dejan una menor huella en el medio ambiente (como las pinturas minerales, y más específicamente, las pinturas a base de cal). En este artículo se describe de forma breve la historia de este material para luego definir la diferencia entre una pintura a la cal moderna y una pintura de cal tradicional. A continuación, se repasan las normas para saber qué exigencias se le pide a este producto tradicional hoy en día. Finalmente, se analizan las fichas técnicas de pinturas a la cal que se comercializan en Europa, en donde se observa que en pocas ocasiones se aplica la clasificación estipulada por la norma EN 1062 1 (EUROPEAN..., 2004) sobre pinturas y barnices y la directiva 2004/42 CE (DIARIO..., 2004).
\end{abstract}

Palablas-clave: Pintura a base de cal. Normativa. Clasificación.

\section{Abstract}

'Brenda Levano ${ }^{1}$ Universitat Politècnica de Catalunya Barcelona - España

${ }^{2}$ Antonia Navarro Ezquerra ${ }^{2}$ Universitat Politècnica de Catalunya Barcelona - España

3Joan Ramon Rosell Amigó ${ }^{3}$ Universitat Politècnica de Catalunya Barcelona - España

Recebido em 14/12/17 Aceito em 08/06/18
In the late 1970's, society started to be aware of the health and environmental risks posed by synthetic paints. Those issues motivated a search for traditional "healthy" products that would leave a smaller environmental footprint (like mineral paints, such as lime-based paints). This study briefly describes the history of this material, and thereafter, it defines the difference between modern limebased paints and traditional lime-based paints. Subsequently, a presents a review of the regulations is presented in order to find out which requirements this traditional product must meet in the present day. Finally, the study analyses technical data sheets of some brands of modern lime-based paints that are sold in Europe. In this analysis we noticed that very few paints are classified according to the EN 1062-1 (EUROPEAN..., 2004) standard for paints and varnishes and the 2004/422 Directive (DIARIO..., 2014).

Keywords: Lime-based paint. Standard. Classification.

LEVANO, B.; NAVARRO, A.; ROSSEL, J. R. Pinturas a base de cal: revisión del mercado y acotación normativa. Ambiente75 Construído, Porto Alegre, v. 18, n. 4, p. 75-83, out./dez. 2018. 


\section{Antecedentes}

La datación más antigua del uso de la cal en revestimientos es de aproximadamente los años 6600-5650 a.C. en Anatolia, específicamente en el asentamiento de Çatalhöyük (Figura 1), en donde podían verse pilares de madera recubiertos con una mezcla de cal pintada de rojo (MELLART, 1971).

Entre los años 2000 y 1400 a.C., en el palacio de Knosos, se hallaron los ejemplares más antiguos de pintura al fresco. Este estilo creció y se desarrolló en la época griega y romana. Por otra parte, la pintura a base de cal (encalados y veladuras de cal) comenzó a popularizarse en Europa en la Edad Media por sus propiedades desinfectantes, transpirables e ignífugas, usándosela mayormente en el exterior de las casas, cuadras, barracones y cuarteles. Su uso en la ciudad se extendió hasta principios de 1900 , mientras que en las zonas rurales se la empleó hasta mitad del siglo $\mathrm{XX}$.

En ese mismo siglo la pintura sintética fue ganando rápidamente el terreno de las pinturas tradicionales gracias a su facilidad de aplicación, la amplia gama de colores y el precio reducido. Sin embargo, décadas más tarde la reputación de las primeras se vio afectada por los problemas de salud y medioambientales que ocasionaban sus componentes (metales pesados y componentes orgánicos volátiles $[\mathrm{COV}])$. Su uso se asoció al cáncer (BROWN et al., 2002), el asma y la leucemia infantil, además de daños cerebrales (GREENSPEC, 2015), problemas pulmonares (MENDELL, 2007), dolores de cabeza, mareos y debilitamiento muscular (AMERICAN LUNG..., 2015).

La creciente concienciación social sobre estos temas ha hecho que se creen normas que prohíban el uso de materiales tóxicos y que se reduzca el contenido de COV. Este hecho, junto con el auge de la construcción ecológica, ha estimulado tanto a fabricantes como a usuarios a buscar "nuevas" alternativas sostenibles, una de ellas son las pinturas tradicionales, como las hechas a base de cal.

\section{Definición y técnicas de aplicación}

Se define como "pintura a base de cal" a la pintura conformada por:

(a) un conglomerante de cal que puede ser aéreo o hidráulico, siendo este el componente que condiciona en mayor medida el comportamiento de la pintura;

(b) pigmentos de tierras naturales (como máximo, $25 \%$ del peso de la cal) u óxidos de hierro (como máximo, $15 \%$ del peso de la cal);

(c) agua o agua de cal, como disolvente; y

(d) aditivos (sólo si son necesarios).

Las técnicas de aplicación que engloba este tipo de pintura son dos:

(a) técnica al fresco o buon fresco, en la que los pigmentos son diluidos en agua de cal y se integran en un enlucido cuando este aún está fresco;

(b) técnica al seco, en la que la pintura se aplica sobre un sustrato seco de origen mineral.

Dependiendo de la proporción de aglutinante y disolvente (Tabla 1), el tipo de pintura puede recibir el nombre de veladura, pintura o encalado o enjalbegado.

Actualmente las pinturas a base de cal que se encuentran en el mercado no son las mismas que se manufacturaban tradicionalmente. Los requerimientos que hoy exigen los usuarios son otros como, facilidad de aplicación, buena adherencia a una gran variedad de sustratos, amplia gama de colores, estabilidad cromática, etc. Esto ha llevado a que los fabricantes le añadan componentes orgánicos a las formulaciones tradicionales.

Para diferenciar a esta nueva generación de pinturas a base de cal de las tradicionales se proponen las denominaciones "pintura a la cal" y "pintura de cal", ambas presentadas a continuación.

Pintura a la cal: se trata de una pintura compuesta por cal, agua o agua de cal, tierras naturales $u$ óxidos de hierro y aditivos orgánicos cuya dosificación y contenido de COV no sobrepase lo indicado por parte de la normativa vigente.

Pintura de cal: pintura comúnmente producida de forma artesanal y compuesta por cal, agua o agua de cal, pigmentos naturales como óxidos de hierro (colores cálidos, no orgánicos) y azul índigo (colores fríos, orgánicos), además de aditivos no orgánicos (Figuras 2a y 2b).

Algunas de las propiedades de la pintura de cal se presentan en el Cuadro 1.

Las Figuras 3a y $3 \mathrm{~b}$ muestran las capas superpuestas de pintura a la cal en la ciudad de Medina de Tetuán, en Marruecos. 
Figura 1 - Reconstrucción del interior de una vivienda de Çatalhöyük

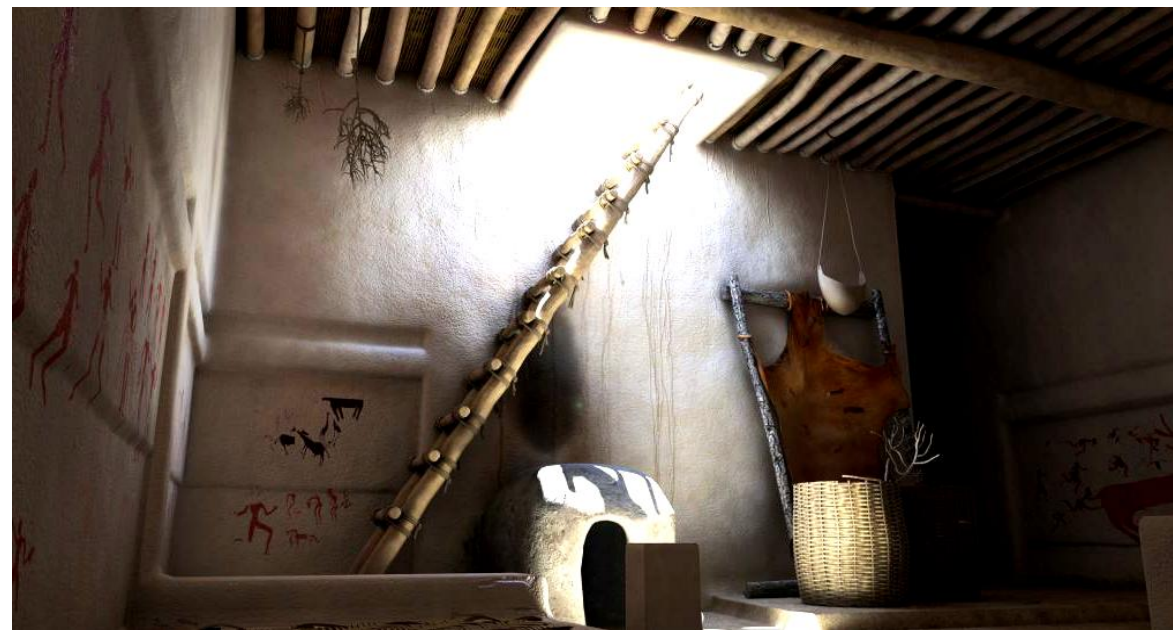

Fuente: Cox (2017).

Tabla 1 - Volumen de cal y de disolvente para distintos tipos de pintura a la cal

\begin{tabular}{l|c|c}
\hline $\begin{array}{c}\text { Tipo de pintura a base } \\
\text { de cal }\end{array}$ & $\begin{array}{c}\text { Volumen } \\
\text { de cal }\end{array}$ & $\begin{array}{c}\text { Volumen de agua o } \\
\text { de agua de cal }\end{array}$ \\
\hline Veladura & $1 \mathrm{~L}$ & $7-30 \mathrm{~L}$ \\
Pintura & $1 \mathrm{~L}$ & $4-7 \mathrm{~L}$ \\
Encalado o enjalbegado & $1 \mathrm{~L}$ & $2-3 \mathrm{~L}$ \\
\hline
\end{tabular}

Fuente: Rattazzi (2007).

Figura 2 - Cal viva y pigmentos de óxido de hierro en mercado de Medina de Tetuán, Marruecos

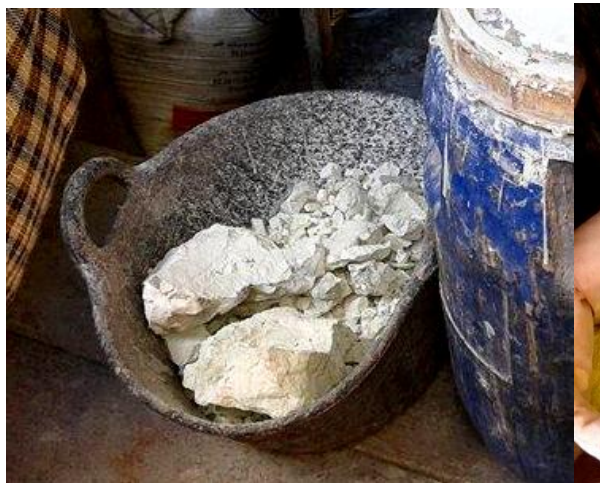

(a)

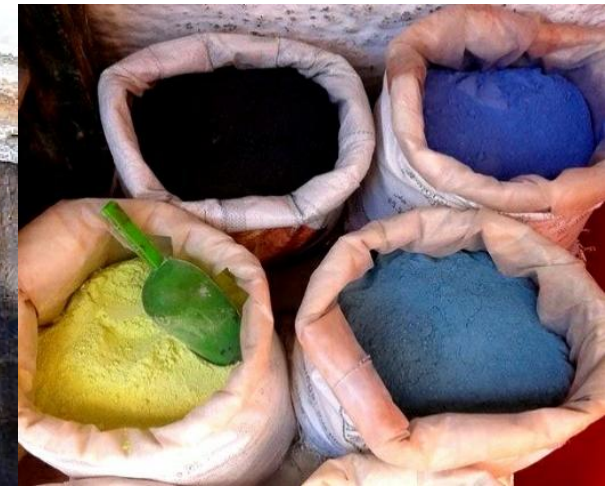

(b)

Cuadro 1 - Propiedades de la pintura de cal artesanal

\begin{tabular}{|l|l|}
\hline \multicolumn{1}{|c|}{$\begin{array}{c}\text { Factor de resistencia al } \\
\text { vapor de água } \boldsymbol{\mu}\end{array}$} & $4-10 \mu$ si no se agrega un aditivo impermeabilizante. \\
\hline Alcalinidad & $\mathrm{pH} 12-13$ \\
\hline Adherencia & $\begin{array}{l}\text { Cohesiva en sustratos minerales incluyendo antiguas capas de pintura de } \\
\text { cal (Figuras } 3 \mathrm{a} \text { y } 3 \mathrm{~b} \text { ). Al carbonatarse, la pintura se acopla a la } \\
\text { superficie mineral y se petrifica creando una estructura microcristalina. }\end{array}$ \\
\hline Acción biocida & $\begin{array}{l}\text { Presente debido a su alta alcalinidad. Esta propiedad dura hasta el final } \\
\text { del proceso de carbonatación }\end{array}$ \\
\hline
\end{tabular}

Fuente: Rattazzi (2007). 
Figura 3 - Capas superpuestas de pintura a la cal en Medina de Tetuán, Marruecos

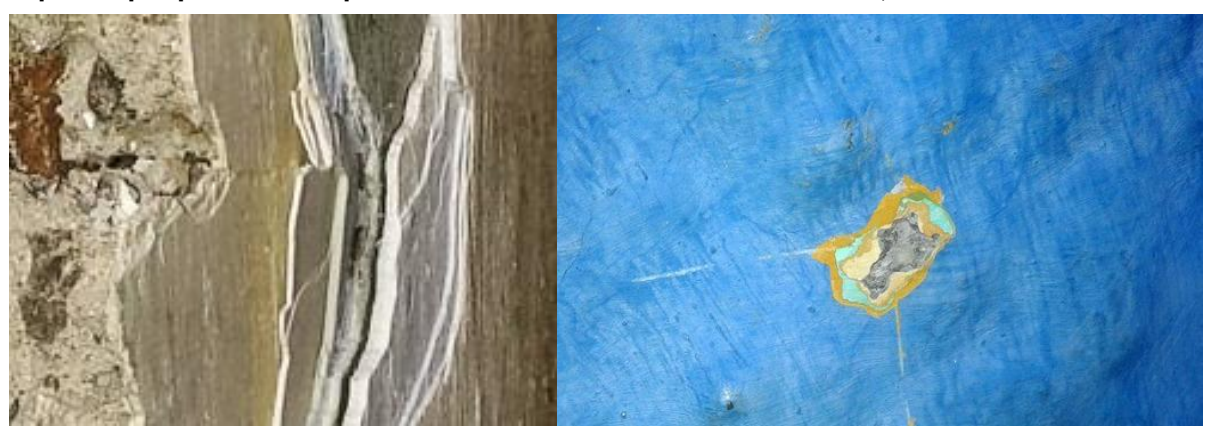

(a)

(b)

Figura 4 - Casas pintadas con pintura de cal en Medina de Tetuán, Marruecos

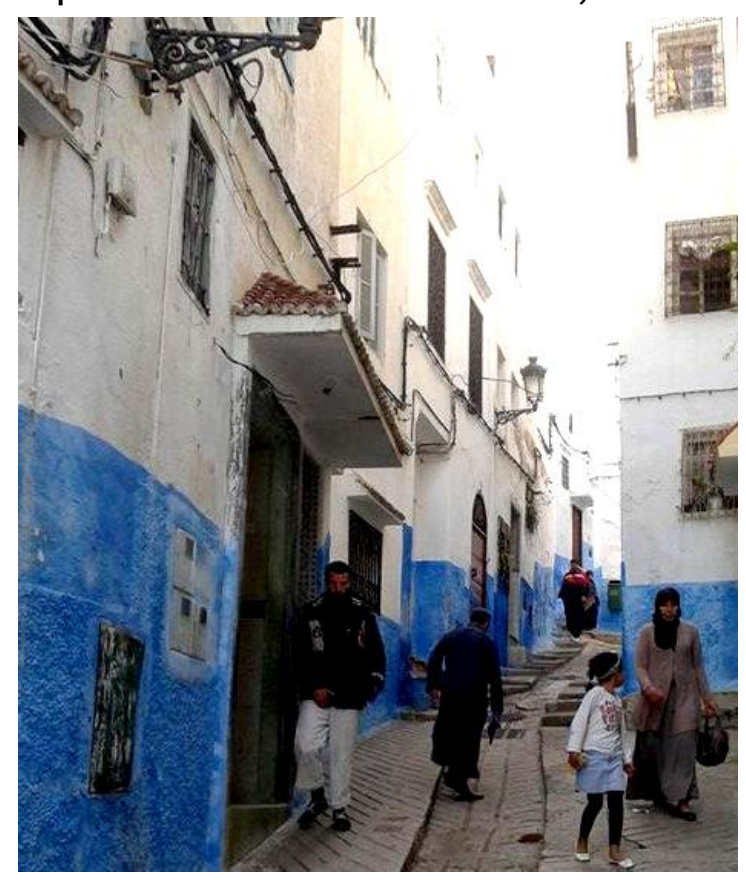

Otras particularidades de la pintura de cal son:

(a) pigmentación variable de acuerdo con la fuerza que aplica el pintor en cada brochazo (como se observa en las Figuras $3 b$ y 4 ) - esta característica se aprovecha para crear profundidad y vibración en las superficies en las que se aplica la pintura;

(b) color de la pintura hasta un 50\% más claro cuando se seca;

(c) cuando fresca, la pintura tiende a decantarse en el recipiente que la contiene, por lo que necesita ser constantemente batida a la hora de su aplicación; y

(d) dependiendo de la densidad de la pintura, son necesarias de 2 a 8 capas para cubrir completamente una superficie.

\section{Normativa sobre pinturas y pinturas a la cal}

Con el fin de profundizar más en el concepto actual de la pintura a la cal y de conocer sus características mínimas, se hizo una búsqueda sobre las normativas relacionadas al tema.

Se encontró que, actualmente, existen tres normas a nivel europeo que las pinturas deben cumplir: dos de clasificación, ya sea para pinturas de interior o de exterior, y una de limitación de emisiones de compuestos orgánicos (Cuadro 2).

Pese a que las normas EN 13300 (EUROPEAN..., 2002) y 1062-1 (EUROPEAN..., 2004) presentan una lista de características que ese debe definir para identificar una pintura, no indica los valores mínimos necesarios para validar este producto. En la búsqueda de reglamentos que proporcionaran

78 Levano, B.; Navarro, A.; Rossel, J. R. 
estos valores mínimos para la pintura a la cal, solo se encontró la norma francesa FDT 30808 (ASSOCIATION..., 2015), que acota la cantidad máxima de componentes orgánicos que puede tener este tipo de pintura $(\leq 5 \%$ de su masa) y proporciona una clasificación según la norma NFT 36-005 (ASSOCIATION..., 2010) que se puede observar en el Cuadro 3.

Desafortunadamente esta norma no ayuda a concretar ningún aspecto de la clasificación según la norma EN 1062-1 (EUROPEAN..., 2004). Entonces, ¿cómo es posible determinar cuál pintura a la cal es apta y cuál no lo es?, ¿se están siguiendo los estándares de una pintura sintética o los de otro tipo de pintura mineral, como la pintura al silicato?

Para responder alguna de estas cuestiones, se analizaron 22 fichas técnicas de pinturas a la cal que se comercializan en Europa, más concretamente en España, Italia, Francia y Alemania (Figura 5). Con ellas se fabricó una serie de tablas que indican las tendencias que está siguiendo este mercado.

Una de las características que se estudió es si las pinturas seguían las normas de clasificación y declaración de la cantidad de COV. Además, también se recogieron otras características que daban a conocer en sus fichas técnicas con más frecuencia. Para resolver esas dudas se creó la Tabla 2. Las características necesarias para la clasificación de la pintura según la norma EN 1062-1 (EUROPEAN..., 2004) están resaltadas en gris.

Las 22 pinturas analizadas podrían aplicarse tanto en el interior como en el exterior, por lo que deberían cumplir la norma de clasificación más estricta, que es la EN 1062-1 (EUROPEAN..., 2004) para exterior. Del total de fichas técnicas analizadas, sólo una presenta la clasificación completa según indica la norma para exteriores. Este producto, originario de Italia, sólo hace referencia a esta norma, puesto que no puede comparar los resultados con otra normativa específica sobre pintura a la cal.

Con respecto al cumplimiento del reglamento 2004/42/CE (DIARIO..., 2004), el 50\% de las fichas analizadas cumple con informar acerca del contenido de COV ( $<30 \mathrm{~g} / \mathrm{L})$. Dado que la mayoría de las pinturas es de origen francés, al cumplir esta norma, también están cumpliendo con la limitación propuesta FDT 30808 (ASSOCIATION..., 2015) del $5 \%$ de la masa.

Cuadro 2 - Normativa referente a las pinturas en general

\begin{tabular}{|l|l|l|l|}
\hline & \multicolumn{1}{|c|}{ Norma } & \multicolumn{1}{c|}{ Nombre } & \multicolumn{1}{c|}{ Descripción } \\
\hline \multirow{2}{*}{$\begin{array}{l}\text { Clasifica- } \\
\text { ción e } \\
\text { identifica- } \\
\text { ción }\end{array}$} & $\begin{array}{l}\text { EN 13300 } \\
\text { EUROPEAN..., 2002) }\end{array}$ & $\begin{array}{l}\text { Materiales y sistema de } \\
\text { recubrimiento en fase acuosa para } \\
\text { paredes y techos interiores. } \\
\text { Clasificación. }\end{array}$ & $\begin{array}{l}\text { Indica un sistema general para } \\
\text { clasificar e identificar una } \\
\text { pintura de interior. }\end{array}$ \\
\cline { 2 - 5 } & $\begin{array}{l}\text { EUROPEAN..., } \\
\text { 2004) }\end{array}$ & $\begin{array}{l}\text { Materiales de recubrimiento y } \\
\text { sistemas de recubrimiento para } \\
\text { albañilería exterior y hormigón. } \\
\text { Parte I: Clasificación. }\end{array}$ & $\begin{array}{l}\text { Indica un sistema general para } \\
\text { clasificar e identificar una } \\
\text { pintura de interior. }\end{array}$ \\
\hline $\begin{array}{l}\text { Normati- } \\
\text { va medio- } \\
\text { ambiental }\end{array}$ & $\begin{array}{l}\text { 2004/42/CE } \\
\text { (DIARIO..., 2004) }\end{array}$ & $\begin{array}{l}\text { Directiva del parlamento Europeo } \\
\text { relativa a la limitación de las } \\
\text { emisiones de compuestos } \\
\text { orgánicos volátiles (COV) } \\
\text { debidas al uso de disolventes } \\
\text { orgánicos en determinadas } \\
\text { pinturas y barnices y en los } \\
\text { productos de renovación del } \\
\text { acabado de vehículos. }\end{array}$ & $\begin{array}{l}\text { Limita la cantidad de COV: } \\
\text { para una pintura de exteriores } \\
\text { de clase "a", el máximo es de } \\
\text { 30 g/L y para una pintura de } \\
\text { tipo "c" con tipología BA el } \\
\text { máximo es de 40g/L. }\end{array}$ \\
\hline
\end{tabular}

Cuadro 3 - Clasificación de la pintura a la cal según la norma FDT 30808:2015y NFT 36-005:2010

\begin{tabular}{|c|l|}
\hline FDT 30808:2015 & \multicolumn{1}{c|}{ NFT 36-005:2010 } \\
\hline Familia I - Categoría 1c & $\begin{array}{l}\text { Familia I: Pinturas y barnices; } \\
\text { Categoría 1: Pintura al agua; } \\
\text { c: pintura a la cal. }\end{array}$ \\
\hline Familia IV - Categoría 4 & $\begin{array}{l}\text { Familia IV: selladores y otros recubrimientos; } \\
\text { Categoría 4: recubrimiento de pintura. }\end{array}$ \\
\hline
\end{tabular}


Figura 5 - Pinturas a la cal estudiadas en España, Italia, Francia, y Alemania

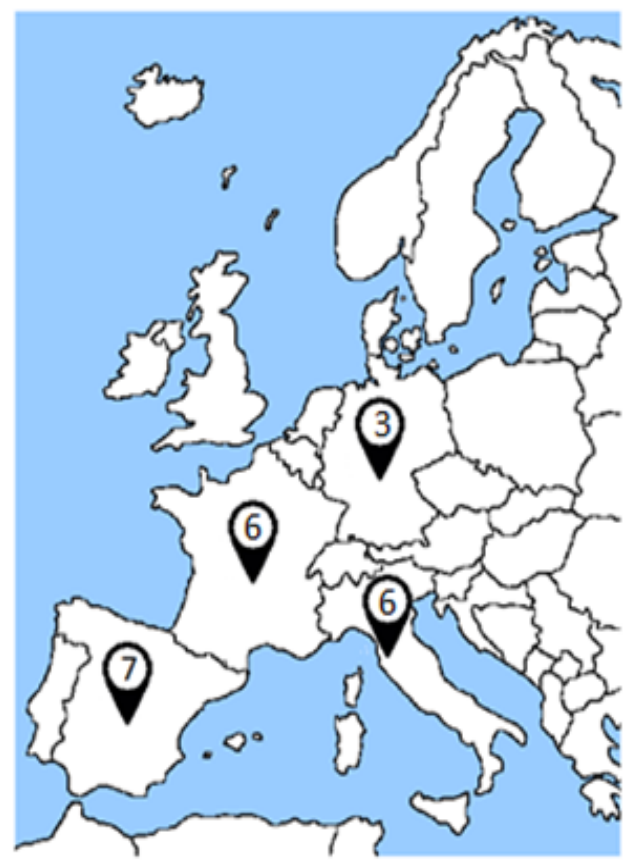

ESPAÑA

- Revetón

- Ecocal

- Hispa Cal

- Cal Lisa

- Calç pint

- Ibercal

- Naturcal

FRANCIA

- Color rare

- Maison Déco

- Tollens

- Corical

- C\&M

- Weber Saint-Gobain $\underline{\text { ITALIA }}$

- PC 144

- San Marco

- La calce del Brenta

- Chiraema

- CVR

- Colorifizio

veneziano

\section{ALEMANIA}

- Kreidezeit

- Auro

- Kalkfarben-Auro

Tabla 2 - Lista de características definidas en las fichas técnicas

\begin{tabular}{l|l|c|c|c|c|c}
\hline \multirow{2}{*}{ Característica } & \multirow{2}{*}{ Rango de resultados } & \multicolumn{3}{c|}{ Repeticiones por país* } & \multirow{2}{*}{ Total** } \\
\cline { 3 - 5 } & & ESP & IT & FR & AL & \\
\hline Rendimiento & $4-10 \mathrm{~m}^{2} / \mathrm{L}$ & $2 / 7$ & $5 / 6$ & $6 / 6$ & $0 / 3$ & $13 / 22$ \\
\hline Peso específico & $1,25-1,67 \mathrm{~kg} / \mathrm{L}$ & $5 / 7$ & $4 / 6$ & $2 / 6$ & $1 / 3$ & $12 / 22$ \\
\hline $\mathrm{pH}$ & $12-13$ & $5 / 7$ & $1 / 6$ & $4 / 6$ & $1 / 3$ & $11 / 22$ \\
\hline Brillo & $<10 \mathrm{a} 85^{\circ}$ & $3 / 7$ & $3 / 6$ & $3 / 6$ & $1 / 3$ & $10 / 22$ \\
\hline Cantidad de COV & $0,01<30 \mathrm{~g}$ & $0 / 7$ & $2 / 6$ & $5 / 6$ & $0 / 3$ & $7 / 22$ \\
\hline $\begin{array}{l}\text { Índice de transmisión de vapor de } \\
\text { agua }(\mathrm{V})^{* * *}\end{array}$ & $>150 \mathrm{~g} / \mathrm{m}^{2} . \mathrm{d}$ & $1 / 7$ & $2 / 6$ & $2 / 6$ & $1 / 3$ & \multirow{2}{*}{$6 / 22$} \\
\hline Permeabilidad al agua líquida & $0,14-0,4 \mathrm{~kg} /\left(\mathrm{m}^{2} . \mathrm{h}^{1 / 2}\right)$ & $1 / 7$ & $3 / 6$ & $1 / 6$ & $0 / 3$ & $5 / 22$ \\
\hline Extracto seco, material no volátil & $50-68 \%$ & $4 / 7$ & $1 / 6$ & $0 / 6$ & $0 / 3$ & $5 / 22$ \\
\hline Densidad & $1,2 \mathrm{y} 1,3 \mathrm{~g} / \mathrm{cm}^{3}$ & $2 / 7$ & $1 / 6$ & $0 / 6$ & $1 / 3$ & $4 / 22$ \\
\hline Granulometría & $\leq 100 \mu \mathrm{m}$ & $0 / 7$ & $3 / 6$ & $0 / 6$ & $0 / 3$ & $3 / 22$ \\
\hline Viscosidad & $16000-60000 \mathrm{cP}$ & $0 / 7$ & $2 / 6$ & $1 / 6$ & $0 / 3$ & $3 / 22$ \\
\hline Comportamiento ante el fuego & $\mathrm{M} 0-\mathrm{M} 1$ & $1 / 7$ & $0 / 6$ & $1 / 6$ & $0 / 3$ & $2 / 22$ \\
\hline Poder cubriente & $92-100 \%$ & $1 / 7$ & $0 / 6$ & $0 / 6$ & $1 / 3$ & $2 / 22$ \\
\hline Espesor de película & $\mathrm{E} 2(50-100) \mu \mathrm{m}$ & $0 / 7$ & $1 / 6$ & $0 / 6$ & $0 / 3$ & $1 / 22$ \\
\hline Resistencia a la fisuración & - & - & - & - & - & - \\
\hline $\begin{array}{l}\text { Permeabilidad al dióxido de } \\
\text { carbono }\end{array}$ & - & - & - & - & - & - \\
\hline
\end{tabular}

Nota: *Número de fichas en la que cada característica fue mencionada; por ejemplo, de las siete fichas técnicas de pintura a la cal estudiadas en España, solo dos mencionaron cuál es el rendimiento de la pintura; **Número total de fichas técnicas de pintura a la cal de los cuatro países estudiados en las que cada característica es mencionada; por ejemplo, el rendimiento de la pintura solo se menciona en trece de las 22 fichas analizadas de los países de España, Francia, Alemania e Italia; $y^{* * *}$ Masa de vapor de agua $(\mathrm{g})$ que pasa por una superficie determinada $\left(\mathrm{m}^{2}\right)$ durante un periodo de tiempo determinado $(24 \mathrm{~h}=\mathrm{d})$.

80 Levano, B.; Navarro, A.; Rossel, J. R. 
Tabla 3- Comparación entre pintura a la cal, pintura al silicato y pintura sintética

\begin{tabular}{|c|c|c|c|c|}
\hline Característica & Rango de resultados & $\begin{array}{c}\text { Clasificación } \\
\text { según EN 1062- } \\
1 \\
\text { (EUROPEAN... } \\
\text {, 2004) } \\
\end{array}$ & $\begin{array}{c}\text { Límite pintura al } \\
\text { silicato UNE } \\
48312 \\
\text { (ASOCIACIÓN.. } \\
., 2009 \text { ) }\end{array}$ & $\begin{array}{l}\text { Límite } \\
\text { pintura } \\
\text { sintética }\end{array}$ \\
\hline Brillo & $<10$ a $85^{\circ}$ & G3-Mate & G3-Mate & - \\
\hline Espesor de película & $50-100 \mu \mathrm{m}(\mathrm{E} 2)$ & E2 & $\mathrm{E} 1(\leq 50)$ & - \\
\hline Granulometría & $\leq 100 \mu \mathrm{m}(\mathrm{S} 1)$ & S1 & S1 & - \\
\hline $\begin{array}{l}\text { Índice de transmisión } \\
\text { de vapor de agua }(\mathrm{V})^{*}\end{array}$ & $>150 \mathrm{~g} / \mathrm{m}^{2} \cdot \mathrm{d}(\mathrm{V} 1)$ & V1 & V1 & V1 \\
\hline $\begin{array}{l}\text { Permeabilidad al agua } \\
\text { líquida }\end{array}$ & $\begin{array}{l}\text { W3: } \leq 0,1 \mathrm{~kg} /\left(\mathrm{m}^{2} \cdot \mathrm{h}^{1 / 2}\right) \\
\text { W2: } 0,14-0,4 \mathrm{~kg} /\left(\mathrm{m}^{2} \cdot \mathrm{h}^{1 / 2}\right) \\
\text { W0: ningún requisito }\end{array}$ & W2 & W0 & W3 \\
\hline $\begin{array}{l}\text { Resistencia a la } \\
\text { fisuración }\end{array}$ & - & $\mathrm{A} 0 * *$ & A0 & - \\
\hline $\begin{array}{l}\text { Permeabilidad al } \\
\text { dióxido de carbono }\end{array}$ & - & A0 & A0 & - \\
\hline
\end{tabular}

Nota: *Masa de vapor de agua $(\mathrm{g})$ que pasa por una superficie determinada $\left(\mathrm{m}^{2}\right)$ durante un periodo de tiempo determinado $(24 h=d) ; y * A 0:$ ningún requisito.

Figura 6 - Espacio pintado con pintura a la cal de la casa

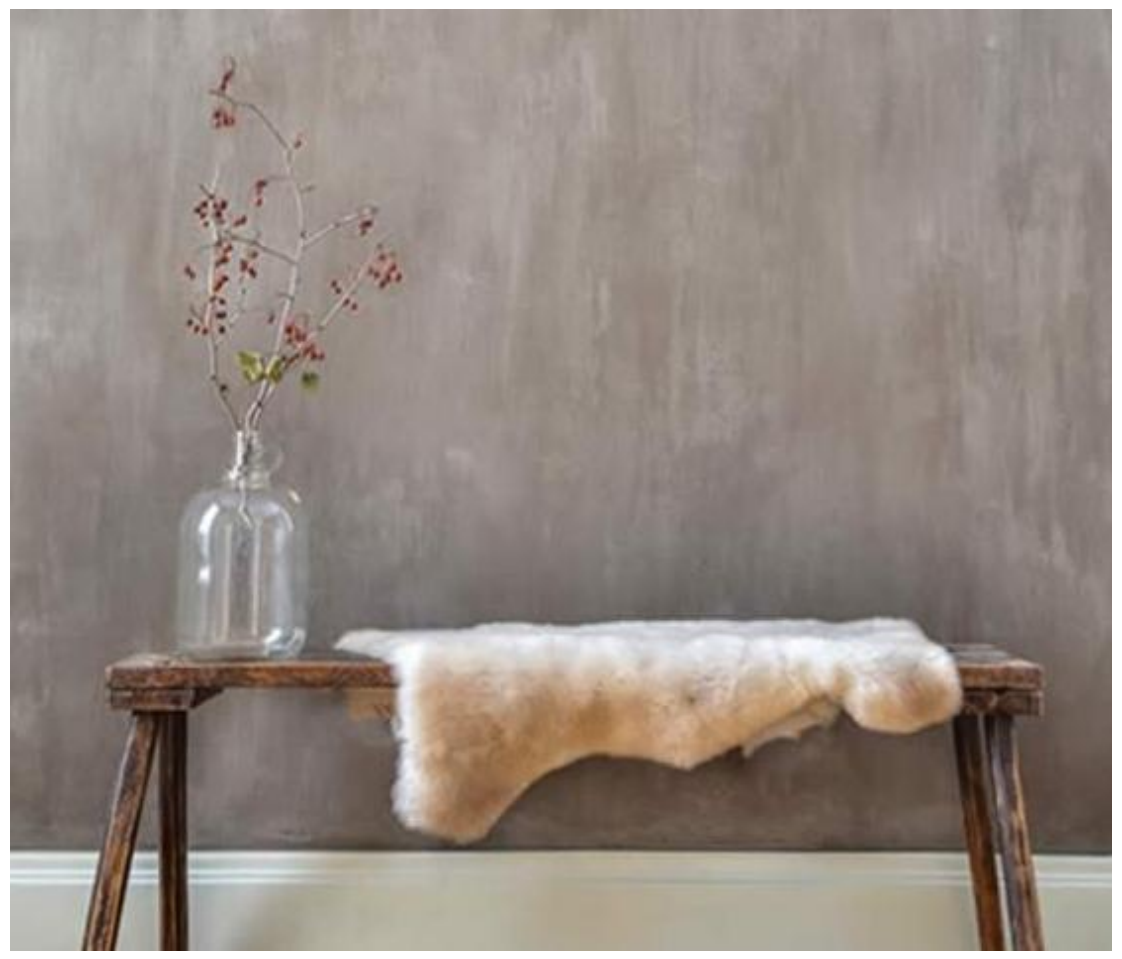

Fuente: Pure \&Original (2017).

Las características que se repiten con más frecuencia en las fichas técnicas son rendimiento, peso específico, $\mathrm{pH}$, brillo y cantidad de $\mathrm{COV}$, las cuales brindan una información fácil de entender para cualquier tipo de consumidor, sea especializado o no.

Con el rango de resultados de la Tabla 2 se puede llegar a identificar la pintura como indica la norma
EN 1062-1 (EUROPEAN..., 2004), para luego compararla con los mínimos que exigen las pinturas al silicato mencionados en la norma UNE 48312 (ASOCIACIÓN..., 2009) y las pinturas sintéticas (Tabla 3).

Como se puede ver, la clasificación obtenida se asemeja más a las restricciones impuestas a la pintura al silicato. Esto se debe a que el 
comportamiento al que hace referencia la norma es bastante similar en los dos tipos de pintura.

Otro aspecto observado en las fichas técnicas fueron las propiedades que estos ofrecían. En todas ellas se mencionaba el brillo de la pintura blanca y los efectos cromáticos y estéticos provocados por el acabado "imperfecto" típico de la pintura de cal (Figura 6), pero además garantizaban la tonalidad de sus colores en todos los lotes de pintura, el aumento del poder cubriente, reduciendo las capas de aplicación a 2, y la mejora de la adherencia en materiales como el yeso, incluso en algunas casas comerciales también podía aplicarse sobre azulejos, mármol, metal y plásticos, sustratos incompatibles para las pinturas de cal tradicionales.

\section{Conclusiones}

Se ha comprobado que la normativa existente sobre pintura a la cal no brinda datos suficientes para acotar y evaluar este producto de manera concluyente. Debido a que es un material cuya demanda, aunque poco a poco, se está incrementando, es pertinente que se cree un reglamento que lo defina de forma apropiada y que, dentro de sus propiedades y limitaciones, diferencie la pintura de cal y la pintura a la cal.

Por otro lado, se observó que la alta transpirabilidad (medida por medio del índice de transmisión de vapor de agua "V") ya no es una propiedad exclusiva de las pinturas minerales, sino que las pinturas sintéticas al agua también están obligadas a cumplirlas. De hecho, se encontró una pintura a la cal que ofrece menor transpirabilidad que una pintura sintética transpirable.

La reducción de esta característica, tan propia de la pintura a base de cal, se debe a la incorporación de material orgánico en su composición. Estos productos se utilizan para mejorar ciertas carencias de la pintura tradicional, como la adherencia al sustrato y el poder aglutinante, lo cual hace que este producto sea más fácil de usar y que tenga una comercialización más estandarizada. Así pues, se debe llegar a un equilibrio entre las distintas características relacionadas con las adiciones orgánicas.

La industrialización de una pintura de cal con las limitaciones que presenta (por ejemplo, en cuanto a la estabilidad del color durante el secado) se podría llevar a cabo si hubiera una mejor difusión y comprensión de su funcionamiento y de sus limitaciones tal como aún se da en ciertos pueblos del mundo, donde el uso de este tipo de pintura sigue estando muy presente.

\section{Referencias}

AMERICAN LUNG ASSOCIATION. Healthy

Air: volatile organic compounds. Disponible en: $<$ http://www.lung.org/our-initiatives/healthyair/indoor/indoor-air-pollutants/volatile-organiccompounds.html?referrer=https://www.google.es/> . Acceso en: 15 dic. 2015.

\section{ASOCIACIÓN ESPAÑOLA DE} NORMALIZACIÓN Y CERTIFICACIÓN. UNE 48312: pintura de silicato en base acuosa para hormigón y albañilería en exteriores. Madrid, 2009.

\section{ASSOCIATION FRANÇAISE DE}

NORMALISATION. FDT 30808: peintures et vernis pour le bâtiment: guide relatif aux produits de peinture et systems de revêtement pour façades.Revêtements minéraux, revêtements organiques. Paris, 2015.

\section{ASSOCIATION FRANÇAISE DE}

NORMALISATION. NFT 36-005: paints and varnishes: characterization of coating materials. Paris, 2010.

BROWN, L. et al. Exposures in the Painting Trades and Paint Manufacturing Industry and Risk of Cancer Among Men and Women in Sweden. Journal of Occupational and Environmental Medicine, Sweden, v. 44, n. 3, p. 258-64, 2002.

COX, G. 3D Artist. Çatalhöyük Shrine of The Hunters Looking South. Disponible en: <http://www.3dartistonline.com/image/11761/atal hyk_shrine_of_the_hunters_looking_south>. Acceso en: 15 dic. 2017.

DIARIO OFICIAL DE LA UNIÓN EUROPEA. Directiva 2004/42/CE, del Parlamento Europeo y del Consejo. 2004.

\section{EUROPEAN COMMITTEE FOR}

STANDARDIZATION. EN 1062-1: paint and varnishes-coating systems for exterior masonry and concrete: part 1: classification. Belgium, 2004.

\section{EUROPEAN COMMITTEE FOR}

STANDARDIZATION. EN 13300: paint and varnishes-water borne coating and coating systems for interior walls and ceilings-classification.

Belgium, 2002.

GREENSPEC. Paint: health \& the environment. Disponible en:

<http://www.greenspec.co.uk/buildingdesign/paint/> .Acceso en: 15 dic. 2015.

MELLAART, J. Earliest Civilizations of the Near East. Ed. McGraw-Hill, 1971. 
MENDELL, M. J. Indoor Residential Chemical Emissions as Risk Factors for Respiratory and Allergic Effects in Children. Berkeley: Indoor Air Journal, 2007.

\section{PURE \& ORIGINAL. The Original Fresco Lime}

Paint color. Disponible en: <http://www.pure-

original.com/photo-gallery/fresco-lime-

paint/fresco-lime-paint-in-the-colour-drift>.

Acceso en: 15 dic. 2017.
RATTAZZI, A. Conosci il Grassello di Calce? Origine, produzione e impiego del grassello in Architettura, nell'arte e nel restauro.

Monfalcone: Edicom, 2007.

Brenda Levano

Master Universitario de Ingeniería de Edificación | Universitat Politècnica de Catalunya | Av. Dr. Marañón 44 | 08028 | Barcelona - España

| E-mail: brenda.levano@hotmail.es

\section{Antonia Navarro Ezquerra}

Laboratori de Materials EPSEB, Departament de Tecnologia del'Arquitectura | Universitat Politècnica de Catalunya | Tel.: +34 (3) 401

6234 | E-mail: antonia.navarro@upc.edu

Joan Ramon Rosell Amigó

Laboratori de Materials EPSEB, Departament de Tecnologia de l'Arquitectura | Universitat Politècnica de Catalunya | E-mail: joan.ramon.rosell@upc.edu

\section{Revista Ambiente Construído}

Associação Nacional de Tecnologia do Ambiente Construído

Av. Osvaldo Aranha, $99-3^{\circ}$ andar, Centro

Porto Alegre - RS - Brasil

CEP $90035-190$

Telefone: +55 (51) 3308-4084

Fax: +55 (51) 3308-4054

www.seer.ufrgs.br/ambienteconstruido

E-mail: ambienteconstruido@ufrgs.br 